phys. stat. sol. (a) 163, 93 (1997)

Subject classification: $68.65 .+\mathrm{g} ; 68.35 . \mathrm{Ct} ; \mathrm{S} 7.13 ; \mathrm{S} 8.13$

\title{
Influence of Growth Parameters on the Surface and Interface Quality of Laser Deposited InSb/CdTe Heterostructures
}

\author{
R. Venkataraghavan (a), K. S. R. K. Rao (a), M. S. Hegde (b), \\ and H. L. BHAT ${ }^{1}$ ) (a) \\ (a) Department of Physics, Indian Institute of Science, Bangalore 560012, India \\ (b) Solid State and Structural Chemistry Unit, Indian Institute of Science, \\ Bangalore 560012, India
}

(Received June 24, 1997)

\begin{abstract}
The pulsed laser deposition technique has been employed for the growth of single crystalline oriented films of indium antimonide on bulk cadmium telluride substrates. The films grown during this study were tested for surface quality and interface features, generally prevalent due to film-substrate reactions. The composition of the grown film was found to deviate from that of the target owing to loss of antimony during evaporation, leading to the formation of an interfacial compound. The antimony deficiency in the films was compensated by correcting the target composition. Growth parameters and their effects on these manifestations have been studied. The optimization of these parameters has led to growth of layers with good surface morphology and abrupt interfaces. Efforts have been made to detect the origin of the diffuse interface and to identify the compound resulting at the interface through photoluminescence spectroscopy. Using PL and thermoenergetic calculations for the plausible reactions at the interface, we have ascertained that the interfacial compound is more likely to be $\operatorname{In}_{2} \mathrm{Te}_{3}$.
\end{abstract}

\section{Introduction}

In the past decade $\mathrm{CdTe} / \mathrm{InSb}$ systems have become prototype for the study of mixed II-VI/III-V heterostructures. In 1984 Welzenis and Ridley [1] theoretically analysed the $\mathrm{InSb} / \mathrm{CdTe} \mathrm{MQW}$ structures and predicted high mobility devices based on indium antimonide. The high band curvature and the small effective mass of the electrons in InSb, leading to extraordinarily high mobilities suited high speed applications in HEMT. This was the first study related to the use of InSb in a heterosystem where it was to play an active and vital role. Apart from offering exciting possibilities of fabricating MQWs and superlattices, the conviction for effective device fabrication is strengthened by their almost perfect lattice match and compatible thermal properties. This led to extensive growth experiments of InSb-CdTe heterostructures with the aim of studying the properties of the MQW. However, all efforts in the fabrication of InSb-CdTe structures have met with only limited success. The growth of $\mathrm{InSb} / \mathrm{CdTe}$, or $\mathrm{CdTe} / \mathrm{InSb}$, has been attached with similar problems, irrespective of the method of growth involved. High annealing temperatures employed in methods like vacuum evaporation $[2,3]$ and temperature gradient vapour transport deposition (TGVTD) [4] have led to diffusive interfaces

1) To whom all correspondence should be addressed. 
in the structure, and hence, the quality of the layers have far belied device standards. The same has been the case with growth by MBE [5 to 9]. OMVPE structures of $\mathrm{CdTe} / \mathrm{InSb}$ by Gandhi and Bhat [10] were found to be of device quality, as probed by photoluminescence spectroscopy, but the report does not comment on the interface. The invariable occurrence of a mixed layer at the film-substrate junction has drawn considerable attention to its study. The compound formed at the interface has been identified as indium telluride $\left(\mathrm{In}_{2} \mathrm{Te}_{3}\right)$ by Kim et al. [4] and independently by Zahn et al. [11, 12] using Raman spectroscopy. However, a report by Welzenis et al. [13], attributes it to a stressed InTe phase.

Laser deposition is relatively a new technique amongst the existing physical vapour deposition methods. Ushered in 1965 with the advances in laser technology, there has been great thrust in the use of this method. The PLD technique is now accepted as a proven method for the growth of high quality multicomponent oxide films. However, until recently, its application to the growth of semiconductor systems has been limited. Photoablation [14], a variant of the PLD technique, has been used as a tool for processing GaAs and InP in a search for low damage processing methods. Mercury cadmium telluride (MCT) epilayers have been successfully grown by laser assisted epitaxy [15]. Zinc selenide (ZnSe), another II-VI wide bandgap semiconductor has also been grown by laser deposition [16]. In fact, superlattices of $\mathrm{ZnSe} / \mathrm{ZnS}$ have been grown by the PLD method by McCamy et al. [17] using hydrogen sulphide as a source gas for ZnS in the PLD chamber. CdTe based solar cells with about 10.5\% AM 1.5 efficiency grown completely by the PLD process have been reported [18]. Though MOCVD and MBE have been considered superior for the growth of semiconductor heterostructures, PLD offers distinct practical advantages, that include stoichiometric material transport and the growth of smooth films. Also, the composition of the various layers can easily be altered by the change of the solid ablation target. This facilitates the growth of strained layer superlattices (SLS) of two or more materials. This clearly indicates that in spite of the lower vacuum levels used in this growth method, device quality layers and heterostructures have been demonstrated. The success of the PLD technique to the production of device quality II-VI structures motivated the present study of InSb/CdTe system through this method. Except for an early attempt to grow InSb by PLD [19], to date there has not been any other report of laser deposited films of this material. In this paper we report the growth of InSb oriented films on CdTe substrates. An in-depth analysis has been made on the interfacial microstructure of this system, looking into ways to improve the interface structure by carefully analysing the role of stoichiometry and identifying the compound present at the interface.

\section{Experimental}

The experimental setup for the laser deposition consisted of a Lambda Physik pulsed excimer laser at $248 \mathrm{~nm}$, which was used to evaporate an InSb polycrystalline disc cast from pre-synthesized compound. The starting materials employed in the present study were $5 \mathrm{~N}$ purity indium and antimony, procured from the Nuclear Fuel Complex Ltd., Hyderabad, India. The substrates used were $\langle 111\rangle$ CdTe wafers, measuring $10 \times 5 \mathrm{~mm}^{2}$, grown and processed at the Solid State Physics Laboratory, Delhi, India. Laser energies of 280 and $100 \mathrm{~mJ} /$ pulse were used for growing two sets of films by laser deposition. The evaporation was carried out in $10^{-4} \mathrm{~Pa}$ vacuum at substrate temperatures ranging from 


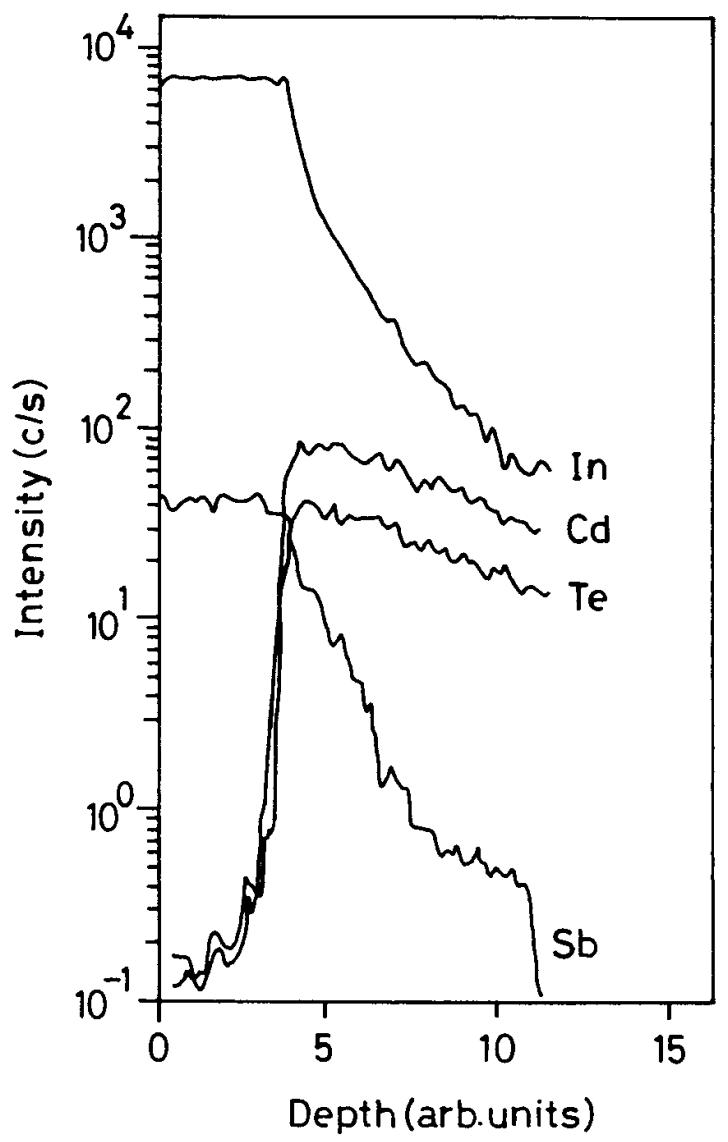

Fig. 1. SIMS profile of the structure from the film into the substrate

185 to $225^{\circ} \mathrm{C}$. Standard experimental techniques like EDAX, SEM, SIMS, X-ray diffraction, IR spectroscopy and optical microscopy were used for various characterizations. Photoluminescence spectra were recorded using a MIDAC FTPL spectrometer at helium temperatures. Laser excitation of $50 \mathrm{~mW}$ were used for recording the spectra, at a resolution of $4 \mathrm{~cm}^{-1}$.

\section{Results and Discussion}

With the view to grow layers with abrupt interfaces and of good surface morphology, initial films were deposited from stoichiometric targets. However, the EDAX of such layers clearly showed that the films grown were off-stoichiometric, in spite of the target being stoichiometric. Also, the interface quality of the films were quite poor, with the film thickness varying by about $\pm 10 \%$ along the length of the interface. The average film thickness based on microscopy was calculated to be around $14 \mu \mathrm{m}$.

In order to get a qualitative measure of the smear at the interface, a SIMS profile was recorded. The profile in Fig. 1 depicting the concentration of $\mathrm{In}, \mathrm{Sb}, \mathrm{Cd}$ and Te along the depth of the film, shows the drop-off of the indium signal, which points to the diffusion of indium into the substrate and the presence of a layer along the interface where $\mathrm{In}, \mathrm{Sb}, \mathrm{Cd}$ and Te coexist. As a result of this it is possible to expect the reaction of the various constituents leading to the formation of an interfacial compound, the presence of which was confirmed by SEM and X-ray diffraction [20].

Inferring that the off-stoichiometry in the films, being one of the reasons for the interfacial reactions, all the subsequent films were grown from a target that contained controlled amounts of excess antimony, that could offset the loss of antimony in the plume during the evaporation. To obtain stoichiometric films, the target composition was optimized by repeated laser deposition experiments and was found to be in the atomic ratio 42.62:57.38 of In:Sb. The films grown from this target were examined for their surface and interface quality. The surface morphology of a typical film grown with the laser energy of $280 \mathrm{~mJ} /$ pulse is shown in Fig. $2 \mathrm{a}$. As can be seen from the figure, the films are 


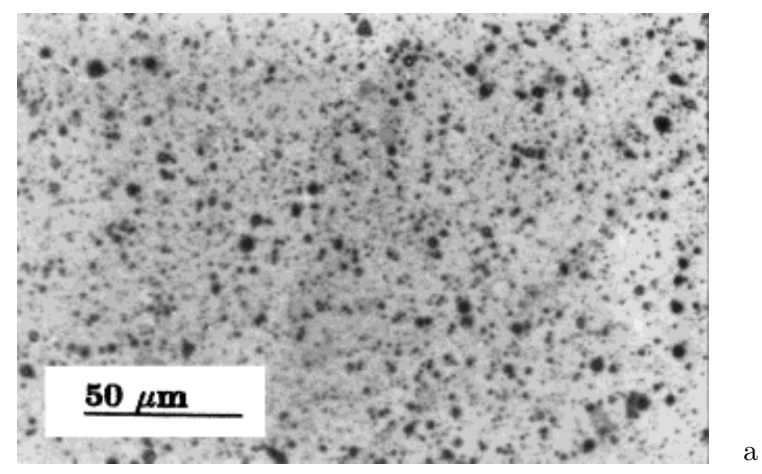

Fig. 2. a) Micrograph showing the pitted morphology of the layers grown at $280 \mathrm{~mJ} /$ pulse; b) micrographs of the surface of the layers grown at $100 \mathrm{~mJ} /$ pulse

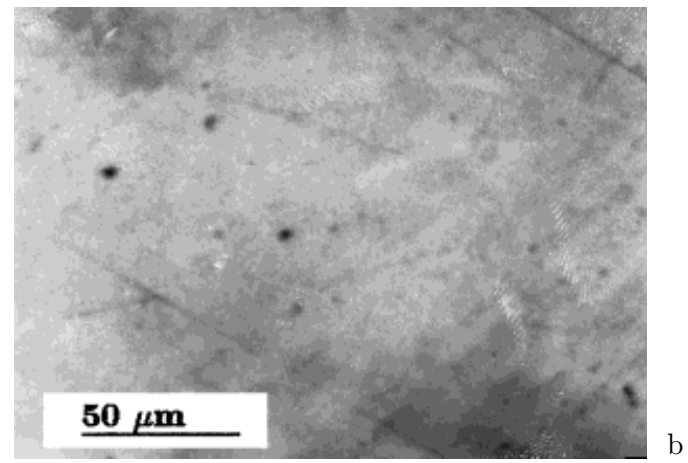

pitted and exhibit a matty surface. This is typical of laser assisted epitaxy where the plume from the target, on getting close to the substrate, heats it, which leads to superheating of the subsurface region of the substrate, thus simultaneously evaporating the substrate surface. The evaporated particles can then get trapped inside the subsurface layer leading to perturbed crystallinity during growth [15]. This leads to a low surface coverage of the substrate and porosity in the grown film. On measuring the resistivity of the films, its value was found to be $83.7 \Omega \mathrm{cm}$ at room temperature. The high resistivity can be attributed to the pitted surface and the porosity in these films.

Since the high energy of the laser beam must have resulted in the poor surface morphology, attempts were made to improve the surface features by reducing the laser power during ablation. This has worked remarkably well, as can be seen in the micrograph (Fig. 2b) of the surface of the film grown at an energy fluence of $100 \mathrm{~mJ} / \mathrm{pulse}$. On measuring the resistivity of these films, the value obtained was a contrasting $1.71 \Omega \mathrm{cm}$ at $300 \mathrm{~K}$, an order of magnitude lower than the values for layers grown at $280 \mathrm{~mJ} /$ pulse.

Although the surface quality could be improved by controlling the laser energy fluence, the interface quality could not be ensured because it does not depend on the composition of the target material alone. Similar manifestations may be seen for off-stoichiometry arising from the substrate. This was realized when stoichiometric films were grown on tellurium rich (cadmium deficient) substrates, as well as on stoichiometric substrates. As-grown CdTe substrates were found to be cadmium deficient, as detected by the presence of a defect luminescence at $1.1 \mathrm{eV}$, attributed to $\mathrm{Cd}$ vacancies, thereby making the substrates tellurium rich. In order to obtain stoichiometric substrates the $\mathrm{Cd}$ 

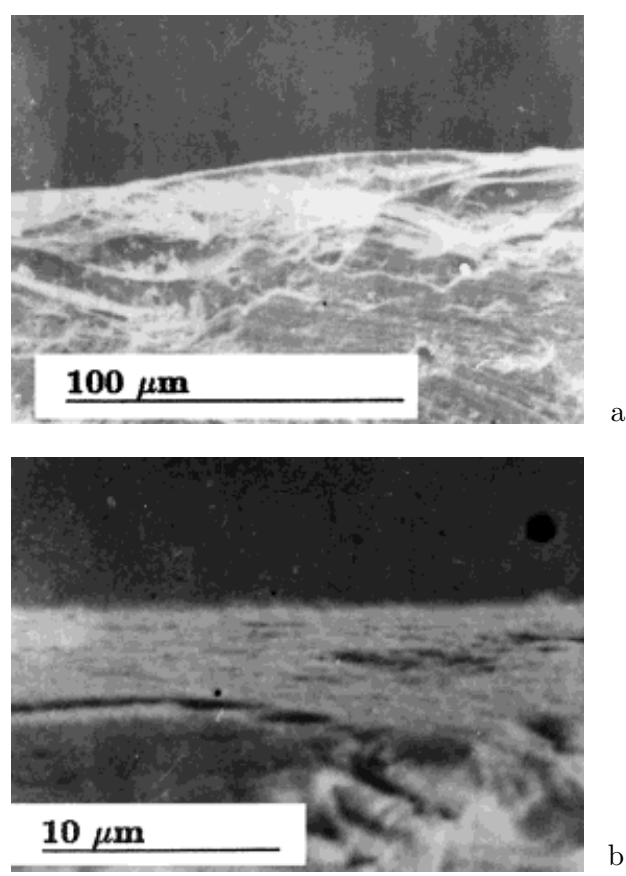

Fig. 3. SEM of the interface of the stoichiometric films grown on a) Te rich CdTe substrates, b) Cd annealed CdTe substrates

deficient crystals were annealed in $\mathrm{Cd}$ overpressures at $650{ }^{\circ} \mathrm{C}$ for $6 \mathrm{~h}$. The removal of cadmium deficiency was verified by recording the PL spectra. The films were then grown on the Te rich and the $\mathrm{Cd}$ annealed substrates to study the dependence of the interface quality on the substrate stoichiometry. The SEM of the interface for films grown on Te rich CdTe substrates (Fig. 3a), and on Cd annealed (stoichiometric) substrates (Fig. 3b), under similar growth conditions, show that the films grown on stoichiometric ( $\mathrm{Cd}$ annealed) substrates are clearly better and do not show a fuzzy interface even at magnifications ten times higher than in the former case. This once again confirms that the interfacial reaction is nothing but a manifestation of the off-stoichiometry in the film or the substrate or in both.

Having established that the interface quality is strongly dependent on the stoichiometry in the layer and the substrate, it was decided to probe the interface in greater detail. There exists divergent views in literature about the chemical nature of the compound at the interface. Although reports of the presence of $\mathrm{In}_{2} \mathrm{Te}_{3}$ at the interface have gained credence owing to two independent researches by Kim et al. [4] and Zahn et al. [11, 12], using Raman spectroscopy, Welzenis et al. [13] have identified the compound to be a stressed InTe phase. Their conclusion is based on Debye-Scherrer X-ray reflection. They have merited the stress in the lattice as a pressure parameter that could alter Raman data and hence produce incorrect results. Wee et al. [21] have used X-ray photoelectron spectroscopy to confirm the presence of $\mathrm{In}_{2} \mathrm{Te}_{3}$ at the interface. Although their results suggest the likelihood of the compound being $\mathrm{In}_{2} \mathrm{Te}_{3}$, due to the matrix effects associated with XPS it was not possible to conclude this with certainty.

In the present work, photoluminescence spectroscopy has been used as a tool to identify the compound at the interface. The various spectra are shown in Fig. 4. The PL spectra recorded for the CdTe substrate usually show a dominant peak at $1.1 \mathrm{eV}$, attributed to the cadmium vacancy and a CdTe signature peak at $1.4 \mathrm{eV}[22]$. The spectrum (a) is that of the InSb/CdTe structure. The $1.1 \mathrm{eV}$ substrate peak appears in this spectra, too, albeit at a reduced intensity in comparison to that from the bare CdTe substrate, due to the screening effect in the film. The samples were then subjected to separate annealings at 150 and $250^{\circ} \mathrm{C}$ for a period of $30 \mathrm{~min}$ each. It is seen that the spectrum recorded after $150^{\circ} \mathrm{C}$ annealing of the sample (spectrum (b)) does not show the $1.1 \mathrm{eV}$ peak which is related to the $\mathrm{Cd}$ vacancy level. This is due to the doping effect of indium (that diffuses from the film into the substrate during the annealing 


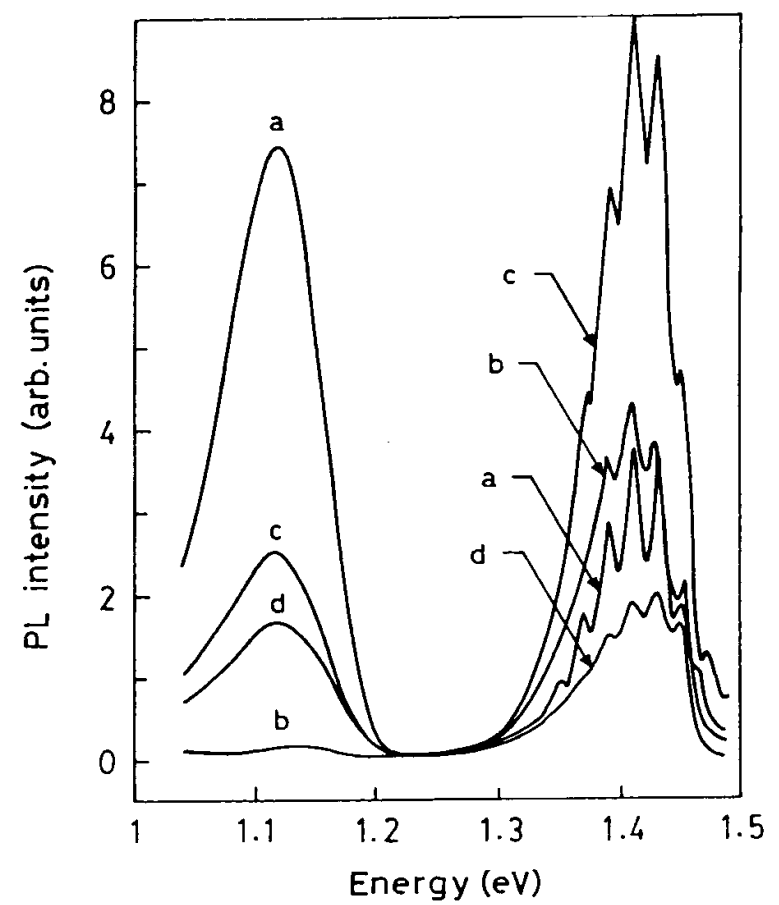

Fig. 4. Photoluminescence spectrum of (a) the InSb/CdTe structure, (b) after $150{ }^{\circ} \mathrm{C}$ anneal, (c) after $250{ }^{\circ} \mathrm{C}$ anneal, and (d) stoichiometric film on Cd annealed substrate

process) resulting in the reduction of the $\mathrm{Cd}$ vacancy concentration in cadmium telluride [22]. However, this peak reappears on annealing at $250{ }^{\circ} \mathrm{C}$ (spectrum (c)). Since it is not possible to generate $\mathrm{Cd}$ vacancies at this temperature, the observed increase in intensity of the $1.1 \mathrm{eV}$ peak on further annealing cannot be attributed to the formation of $\mathrm{Cd}$ vacancies. Hence, the origin of this new peak is not related to the $\mathrm{Cd}$ defect peak, and is likely to be only due to the formation of an interfacial compound whose bandgap lies around $1.1 \mathrm{eV}$. Reports on the bandgap of $\mathrm{In}_{2} \mathrm{Te}_{3}$ range between 1.0 to $1.2 \mathrm{eV}[23,24]$ at room temperature and about $1.2 \mathrm{eV}$ at $0 \mathrm{~K}$ [25] for the bulk. However, for the MBE grown films [26], the bandgap has been found to be $1.3 \mathrm{eV}$ at $77 \mathrm{~K}$ and hence should be even higher at $4.2 \mathrm{~K}$, the temperature at which the spectra are recorded. Considering that the $1.1 \mathrm{eV}$ peak originates from the interface, and the bulk value for the indium telluride bandgap is coincident to this, it can be said that the compound at the interface is likely to be bulk in nature rather than a thin film. Our earlier micrographical studies of the interfacial layer [20] corroborate this view.

The spectrum in (d) of Fig. 4 is that of the Cd annealed substrate over which a stoichiometric InSb film is grown at lower energy fluence. The intensity of the 1.1 and $1.4 \mathrm{eV}$ peaks, due to the substrate and the interface, are clearly negligible. This suggests that these films, grown from the off-stoichiometric target on Cd annealed substrates, are the best in terms of surface morphology and interface quality.

Depending on the conditions expected at the time of growth, various reactions are postulated and their free energies calculated to assess their possibility. This has been used to study the effect of $\mathrm{Cd}$ enhanced fluxes in reducing the interfacial reactivity [7]. Welzenis and coworkers [13] through their calculations indicate the presence of InTe at the interface. Golding and coworkers [27], took into account the kinematics of the vari- 
ous process occurring at the growth front to postulate the exclusion of Te from the interface, implying that Te 'floats' on the InSb growth surface and is not incorporated at the interface. Such a postulate was consistent with their Auger data. This is in variance with our SIMS results which show the presence of all elemental species at the interface. This must be essentially because of the considerable difference in growth rates of the MBE and the PLD techniques. The fastest growth rate as reported by Golding et al. [27] was $0.7 \mu \mathrm{m} / \mathrm{h}$ for the layers. This leaves ample time for the elimination of InTe from the growth 'zone' in the MBE case. However, in our case the average growth rate was $45 \mu \mathrm{m} / \mathrm{h}$ and at this rate it is more likely that the growth process leads to the incorporation of Te at the interface itself.

Under these conditions, it was initially thought that the following reactions may be occurring at the interface:

$$
\begin{array}{ll}
\mathrm{InSb}+\mathrm{CdTe} \rightarrow \mathrm{InTe}+\mathrm{Cd}+\mathrm{Sb} & (\Delta G=45.26 \mathrm{~kJ} / \mathrm{mol}), \\
\mathrm{InSb}+3 / 2 \mathrm{CdTe} \rightarrow 1 / 2 \mathrm{In}_{2} \mathrm{Te}_{3}+3 / 2 \mathrm{Cd}+\mathrm{Sb} & (\Delta G=75.94 \mathrm{~kJ} / \mathrm{mol}) .
\end{array}
$$

The $\Delta G$ values for the reactions are calculated from the known values for the Gibbs energies for the individual reactants [28]. As can be seen, the Gibbs free energy for the above reactions are positive, indicating that none of the reactions is spontaneous. Even on recalculating the Gibbs energies, taking into account the reduced pressures in the growth chamber, the values one gets are 46.29 and $62.94 \mathrm{~kJ} / \mathrm{mol}$, respectively, for these reactions. The changes being negligible, one concludes that even at reduced pressure in the growth chamber, there is no possibility of any aggregate formation. For the above reactions, it has been assumed that both the reactants are molecular in nature. But it has already been seen that the film is rich in indium and the substrate is rich in tellurium. Hence, the more apt reactions to be considered would be the following, by which it is clear that the possibility of the formation of $\mathrm{In}_{2} \mathrm{Te}_{3}$ is greater by virtue of its lower Gibbs energy:

$$
\begin{array}{ll}
\mathrm{In}+\mathrm{Te} \rightarrow \operatorname{InTe} & (\Delta G=-71 \mathrm{~kJ} / \mathrm{mol}), \\
2 \mathrm{In}+3 \mathrm{Te} \rightarrow \mathrm{In}_{2} \mathrm{Te}_{3} & (\Delta G=-177 \mathrm{~kJ} / \mathrm{mol}) .
\end{array}
$$

This also suggests that the occurrence of the interfacial compound is possible only when there is availability of $\mathrm{In}$ and Te in elemental form. Hence, the interfacial compound formation can be minimized by eliminating their excess by strictly monitoring stoichiometry. This is what has been achieved experimentally by us in the stoichiometric films grown on the stoichiometric substrates.

\section{Conclusion}

In conclusion, oriented films of InSb with abrupt interfaces have been grown on bulk CdTe substrates by the pulsed laser deposition technique, at temperatures ranging between 185 and $225^{\circ} \mathrm{C}$. The $\mathrm{InSb} / \mathrm{CdTe}$ heterojunctions pose the problem of interfacial layer formation which could be attributed to the lack of stoichiometry in the grown films and can be controlled by monitoring the stoichiometry. The effects of off-stoichiometric substrates on the film quality have been investigated. Growing the layers at lower temperatures, helps to prevent interdiffusion of the atomic species across the interface leading to aggregate formation at the interface. The compound at the interface has been 
identified as $\mathrm{In}_{2} \mathrm{Te}_{3}$ rather than InTe, based on the results of X-ray diffraction and PL spectroscopy and are further strengthened by thermoenergetic calculations.

Acknowledgements The authors acknowledge the help of Dr. Vikram Kumar, Director, SPL, Delhi for access to the SIMS facility, Mr. Sam Phillip, Material Research Center, IISc, Bangalore for technical assistance with EDAX and SE microscopy, Ms. K. M. Satyalakshmi and Ms. Sahana for carrying out the laser evaporation of the films.

\section{References}

[1] R. G. van Welzenis and B. K. Ridley, Solid State Electronics 27, 113 (1984).

[2] G. LeFloch, Thin Solid Films 2, 383 (1968).

[3] B. Rabin, C. Scharager, M. Hage-Ali, P. Siffert, F. V. Wald, and R. O. Bell, phys. stat. sol. (a) 62, 237 (1980).

[4] T. W. Kim, M. Jung, H. L. Park, H. K. NA, and J. S. Kim, Appl. Phys. Lett. 61, 1101 (1992).

[5] K. Sugiyama, J. Cryst. Growth 60, 450 (1982).

[6] G. M. Williams, C. R. Whitehouse, N. G. Chew, G. W. Blackmore, and A. G. Cullis, J. Vac. Sci. Technol. B 3, 704 (1985).

[7] T. D. Golding, M. Martinka, and J. H. Dinan, J. Appl. Phys. 64, 1873 (1988).

[8] K. J. Mackey, P. M. G. Allen, W. G. Herrenden Harker, and R. H. Williams, Surf. Sci. 178, 124 (1986).

[9] K. J. Mackey, P. M. G. Allen, W. G. Herrenden Harker, R. H. Williams, C. R. Whitehouse, and G. M. Williams, Appl. Phys. Lett. 49, 354 (1986).

[10] S. K. Gandhi and I. Bhat, Appl. Phys. Lett. 45, 678 (1984).

[11] D. R. T. Zahn, K. J. Mackey, R. H. Williams, H. Muder, J. Geurts, and W. Richter, Appl. Phys. Lett. 50, 742 (1987).

[12] D. R. T. Zahn, W. Richter, T. Eickhoff, J. Geurts, T. D. Golding, J. H. Dinan, K. J. Mackey, and R. H. Williams, Appl. Surf. Sci. 41/42, 497 (1989).

[13] R. G. van Welzenis, F. M. van Setten, and O. F. Z. Schannen, Appl. Phys. A 52, 19 (1991).

[14] J. D. Moore, P. R. Herman, and R. A. Potyrailo, AIP Conf. Proc. 288, 77 (1994).

[15] J. T. Cheung and T. Magee, J. Vac. Sci. Technol. A 1, 1604 (1983).

[16] Y. Rajakarunanayake, Y. Luo, B. T. Adkins, X. Dai, and A. Compann, AiP Conf. Proc. 288, 577 (1994).

[17] J. W. McCamy, D. H. Lowndes, and J. D. Budai, Internat. Conf. Solid State Devices and Materials, Tsukuba (Japan), 1992 (p. 357).

[18] A. Compann, C. N. Tabory, M. Shao, Y. Li, A. Fischer, Z. Feng, and R. G. Bohn, AIP Conf. Proc. 288, 225 (1994).

[19] S. V. Gaponov, B. M. Luskin, and N. N. Salaschenko, Soviet Phys. - Semicond. 14, 873 (1980).

[20] R. Venkataraghavan, K. M. Satyalakshmi, K. S. R. K. Rao, A. K. Sreedhar, M. S. HedGe, and H. L. Bhat, Bull. Mater. Sci. 19, 123 (1996).

[21] A. T. S. Wee, Z. C. Feng, H. H. Hng, K. L. Tan, R. F. C. Farrow, and W. J. Choyke, J. Phys.: Condensed Matter 7, 4359 (1995).

[22] S. Gurumurthy, K. S. R. K. RaO, A. K. Sreedhar, H. L. Bhat, B. Sundersheshu, R. K. Bagai, and V. Kumar, Bull. Mater. Sci. 17, 1057 (1994).

[23] S. Ozaki, K. Takada, and S. Adachi, Jpn. J. Appl. Phys. 33, 6213 (1994).

[24] S. Sen and D. N. Bose, Solid State Commun. 50, 39 (1984).

[25] CRC Handbook of Chemistry and Physics, 74th ed., Ed. D. R. Lide, CRC Press, Boca Raton (Florida) 1993/94.

[26] T. D. Golding, P. R. Boyd, M. Martinka, P. M. Amrithraj, J. H. Dinan, S. B. Qadri, D. R. T. Zahn, and C. R. Whitehouse, J. Appl. Phys. 65, 1936 (1989).

[27] T. D. Golding, J. Strozier, J. H. Dinan, and D. Zahn, Mater. Res. Soc. Symp. Proc. 221, 311 (1991).

[28] O. Kancke, O. Kubaschewski, and K. Hesselmann (Eds.), Thermochemical Properties of Inorganic Substances, 2nd ed., Springer-Verlag, Berlin/Heidelberg/New York 1991. 